\title{
Article
}

\section{On a generalized class of bi-univalent functions defined by subordination and $q$-derivative operator}

\author{
Ayotunde O. Lasode ${ }^{1, *}$ and Timothy O. Opoola ${ }^{1}$ \\ 1 Department of Mathematics, University of Ilorin, PMB 1515, Ilorin, Nigeria. \\ * Correspondence: lasode_ayo@yahoo.com \\ Academic Editor: Nak Eun Cho \\ Received: 19 June 2021; Accepted: 25 August 2021; Published: 6 September 2021.
}

\begin{abstract}
In this paper, the $q$-derivative operator and the principle of subordination were employed to define a subclass $\mathcal{B}_{q}(\tau, \lambda, \phi)$ of analytic and bi-univalent functions in the open unit disk $\mathcal{U}$. For functions $f(z) \in \mathcal{B}_{q}(\tau, \lambda, \phi)$, we obtained early coefficient bounds and some Fekete-Szegö estimates for real and complex parameters.
\end{abstract}

Keywords: Analytic function; Bi-univalent function; Subordination; Fekete-Szegö problem; Ma-Minda function; Carathéodory function; $q$-differentiation.

MSC: 30C45; 30C50.

\section{Introduction}

$\mathbf{L}$

et $\mathcal{U}=\{z: z \in \mathbb{C},|z|<1\}$ be a unit disk and let $\mathcal{A}$ denote the class of analytic functions of the form

$$
f(z)=z+\sum_{n=2}^{\infty} a_{n} z^{n} \quad(z \in \mathcal{U}),
$$

normalized by the conditions $f(0)=f^{\prime}(0)-1=0$. Let $\mathcal{S} \subset \mathcal{A}$ be the class of analytic and univalent functions in $\mathcal{U}$.

Let $\mathcal{W}$ denote the class of functions

$$
w(z)=w_{1} z+w_{2} z^{2}+w_{3} z^{3}+\cdots \quad(z \in \mathcal{U})
$$

such that $w(0)=0$ and $|w(z)|<1$. The class $\mathcal{W}$ is known as the class of Schwarz functions.

By [1], let $j(z), J(z) \in \mathcal{A}$, then $j(z) \prec J(z), z \in \mathcal{U}$, if $\exists w(z)$ analytic in $\mathcal{U}$, such that $w(0)=0,|w(z)|<1$ and $j(z)=J(w(z))$. If the function $J(z)$ is univalent in $\mathcal{U}$, then $j(z) \prec J(z) \Longrightarrow j(0)=J(0)$ and $j(\mathcal{U}) \subset J(\mathcal{U})$.

Let $\mathcal{P}$ denote the class of functions

$$
p(z)=1+p_{1} z+p_{2} z^{2}+\cdots \quad(z \in \mathcal{U})
$$

which are analytic in $\mathcal{U}$ such that $\mathcal{R} e(p(z))>0$ and $p(0)=1$. It is known that functions in classes $\mathcal{P}$ and $\mathcal{W}$ are related such that

$$
p(z)=\frac{1+w(z)}{1-w(z)} \Longleftrightarrow w(z)=\frac{p(z)-1}{p(z)+1} .
$$

In [2], Ma and Minda defined a function $\phi \in \mathcal{P}(z \in \mathcal{U})$ such that $\phi(0)=1, \phi^{\prime}(0)>0$ and $\phi(\mathcal{U})$ is starlike with respect to 1 and symmetric with respect to the real axis. Such function $\phi$ can be expressed as

$$
\phi(z)=1+\beta_{1} z+\beta_{2} z^{2}+\cdots \quad\left(z \in \mathcal{U}, \beta_{1}>0\right) .
$$

Fekete and Szegö [3] investigated the coefficient functional

$$
g_{\rho}(f)=\left|a_{3}-\rho a_{2}^{2}\right|
$$


which arose from the disproof of Littlewood-Parley conjecture (see [1]) that says modulus of coefficients of odd univalent functions are less than 1 . This functional has been investigated by many researchers, see for instance $[4,5]$.

Historically, Lewin [6] introduced a subclass of $\mathcal{A}$ called the class of bi-univalent functions and established that $\left|a_{2}\right| \leq 1.51$ for all bi-univalent functions. Also, the Koebe $1 / 4$ theorem (see [1]) states that the range of every function $f \in \mathcal{S}$ contains the disk $D=\{\omega:|\omega|<0.25\} \subseteq f(\mathcal{U})$. This implies that $\forall f \in \mathcal{S}$ has an inverse function $f^{-1}$ such that

$$
f^{-1}(f(z))=z \quad(z \in \mathcal{U})
$$

and

$$
f\left(f^{-1}(\omega)\right)=\omega \quad\left(\omega:|\omega|<r_{0}(f) ; r_{0}(f) \geq 0.25\right),
$$

where $f^{-1}(\omega)$ is expressed as

$$
F(\omega)=f^{-1}(\omega)=\omega-a_{2} \omega^{2}+\left(2 a_{2}^{2}-a_{3}\right) \omega^{3}-\left(5 a_{2}^{3}-5 a_{2} a_{3}+a_{4}\right) \omega^{4}+\cdots .
$$

Thus, a function $f \in \mathcal{A}$ is said to be bi-univalent in $\mathcal{U}$ if both $f(z)$ and $F(\omega)$ are univalent in $\mathcal{U}$. Let $\mathcal{B}$ denote the class of analytic and bi-univalent functions in $\mathcal{U}$.

Some functions $f \in \mathcal{B}$ includes $f(z)=z, f(z)=z /(1-z), f(z)=-\log (1-z)$ and $f(z)=\frac{1}{2} \log [(1+$ $z) /(1-z)]$. Observe that some familiar functions $f \in \mathcal{S}$ such as the Koebe function $K(z)=z /(1-z)^{2}$, its rotation function $K_{\sigma}(z)=z /\left(1-e^{i \sigma} z\right)^{2}, f(z)=z-z^{2} / 2$ and $f(z)=z /\left(1-z^{2}\right)$ are nonmembers of $\mathcal{B}$. See $[4,5,7-11]$ for more details.

Jackson [12] (see also $[8,13,14]$ ) introduced the concept of $q$-derivative operator. For functions $f \in \mathcal{A}$, the $q$-derivative of $f$ can be defined by

$$
\mathcal{D}_{q} f(z)=\frac{f(z)-f(q z)}{(1-q) z} \quad(z \neq 0,0<q<1),
$$

where $\mathcal{D}_{q} f(0)=f^{\prime}(0)$ and $\mathcal{D}_{q} f(z) z=\mathcal{D}_{q}\left(\mathcal{D}_{q} f(z)\right)$. From (1) and (6) we get

$$
\left.\begin{array}{l}
\mathcal{D}_{q} f(z)=1+\sum_{n=2}^{\infty}[n]_{q} a_{n} z^{n-1} \\
\mathcal{D}_{q} f(z) z=\sum_{n=2}^{\infty}[n]_{q}[n-1]_{q} a_{n} z^{n-2}
\end{array}\right\}
$$

where $[n]_{q}=\frac{1-q^{n}}{1-q},[n-1]_{q}=\frac{1-q^{n-1}}{1-q}, \lim _{q \uparrow 1}[n]_{q}=n$ and $\lim _{q \uparrow 1}[n-1]_{q}=n-1$.

For instance, if $\alpha$ is a constant, then for the function $f(z)=\alpha z^{n}$,

$$
\mathcal{D}_{q} f(z)=\mathcal{D}_{q}\left(\alpha z^{n}\right)=\frac{1-q^{n}}{1-q} \alpha z^{n-1}=[n]_{q} \alpha z^{n-1},
$$

and note that

$$
\lim _{q \uparrow 1} \mathcal{D}_{q} f(z)=\lim _{q \uparrow 1}[n]_{q} \alpha z^{n-1}=n \alpha z^{n-1}=: f^{\prime}(z),
$$

where $f^{\prime}(z)$ is the classical derivative.

In this study, the $q$-derivative operator and the subordination principle are used to define and generalize a subclass of bi-univalent functions. Afterwards, some coefficient bounds and some Fekete-Szegö estimates were investigated. Some of our results generalised that of Srivastava and Bansal in [10] and some new results are added. 
Definition 1. Let $0<q<1, \tau \in \mathbb{C} \backslash\{0\}, 0 \leq \lambda \leq 1$ and $\phi$ is defined in (4). A function $f \in \mathcal{B}$ is said to be in the class $\mathcal{B}_{q}(\tau, \lambda, \phi)$ if the subordination conditions

$$
1+\frac{1}{\tau}\left[\mathcal{D}_{q} f(z)+\lambda z \mathcal{D}_{q} f(z) z-1\right] \prec \phi(z) \quad(z \in \mathcal{U}),
$$

and

$$
1+\frac{1}{\tau}\left[\mathcal{D}_{q} F(\omega)+\lambda \omega \mathcal{D}_{q}^{2} F(\omega)-1\right] \prec \phi(\omega) \quad(\omega \in \mathcal{U}),
$$

where $F(\omega)=f^{-1}(\omega)$ are satisfied.

Remark 1. Let $q \uparrow 1$ in (8) and (9), then $\mathcal{B}_{q}(\tau, \lambda, \phi)$ becomes the class $\mathcal{B}(\tau, \lambda, \phi)$ investigated by Srivastava and Bansal [10].

\section{Preliminary Lemmas}

To establish our results, we shall need the following lemmas. Let $p(z)$ be as defined in (2).

Lemma 2 ([1]). If $p(z) \in \mathcal{P}$, then $\left|p_{n}\right| \leq 2(n \in \mathbb{N})$. The result is sharp for the well-known Möbius function.

Lemma $3([15,16])$. If $p(z) \in \mathcal{P}$, then $2 p_{2}=p_{1}^{2}+\left(4-p_{1}^{2}\right) x$ for some $x$ and $|x| \leq 1$.

\section{Main Results}

Unless otherwise mentioned in what follows, we assume throughout this work that $0<q<1, \tau \in$ $\mathbb{C} \backslash\{0\}, 0 \leq \lambda \leq 1, \phi$ is as defined in (4) and $f \in \mathcal{B}$, hence our results are as follows:

Theorem 4. Let $f \in \mathcal{B}_{q}(\tau, \lambda, \phi)$, then

$$
\begin{aligned}
& \left|a_{2}\right| \leq \frac{\beta_{1}^{3 / 2}|\tau|}{\sqrt{\left|\beta_{1}^{2} \tau[3]_{q}\left(1+[2]_{q} \lambda\right)+[2]_{q}^{2}\left(1+[1]_{q} \lambda\right)^{2}\left(\beta_{1}-\beta_{2}\right)\right|}}, \\
& \left|a_{3}\right| \leq \frac{\beta_{1}^{2}|\tau|^{2}}{[2]_{q}^{2}\left(1+[1]_{q} \lambda\right)^{2}}+\frac{\beta_{1}|\tau|}{[3]_{q}\left(1+[2]_{q} \lambda\right)},
\end{aligned}
$$

where $\beta_{1}>0$ and $\beta_{n}(n \in \mathbb{N})$ are coefficients of $\phi(z)$ in (4).

Proof. Let $f(z) \in \mathcal{B}$ and $F(\omega)=f^{-1}(\omega)$, then there exists the analytic functions $u(z), v(\omega) \in \mathcal{W}, z, \omega \in \mathcal{U}$ such that $u(0)=0=v(0),|u(z)|<1,|v(\omega)|<1$ so that they satisfy the subordination conditions:

$$
1+\frac{1}{\tau}\left[\mathcal{D}_{q} f(z)+\lambda z \mathcal{D}_{q} f(z) z-1\right]=\phi(u(z)) \quad(z \in \mathcal{U}),
$$

and

$$
1+\frac{1}{\tau}\left[\mathcal{D}_{q} F(\omega)+\lambda \omega \mathcal{D}_{q}^{2} F(\omega)-1\right]=\phi(v(\omega)) \quad(\omega \in \mathcal{U}) .
$$

By substituting (7) into LHS of (12) we respectively get

$$
1+\frac{1}{\tau}\left[\mathcal{D}_{q} f(z)+\lambda z \mathcal{D}_{q} f(z) z-1\right]=1+\frac{[2]_{q}\left(1+[1]_{q} \lambda\right) a_{2}}{\tau} z+\frac{[3]_{q}\left(1+[2]_{q} \lambda\right) a_{3}}{\tau} z^{2}+\cdots,
$$

and following the same process for $F(\omega)$ in (5) gives

$$
1+\frac{1}{\tau}\left[\mathcal{D}_{q} F(\omega)+\lambda \omega \mathcal{D}_{q}^{2} F(\omega)-1\right]=1-\frac{[2]_{q}\left(1+[1]_{q} \lambda\right) a_{2}}{\tau} \omega+\frac{[3]_{q}\left(1+[2]_{q} \lambda\right)\left(2 a_{2}^{2}-a_{3}\right)}{\tau} \omega^{2}+\cdots
$$

Now to expand

$$
\phi(u(z))
$$


and

$$
\phi(v(\omega)),
$$

in series form, let $\delta_{1}(z)=1+b_{1} z+b_{2} z^{2}+\ldots, \delta_{2}(\omega)=1+c_{1} \omega+c_{2} \omega^{2}+\cdots \in \mathcal{P}$, then by (3),

$$
\delta_{1}(z)=\frac{1+u(z)}{1-u(z)} \Longrightarrow u(z)=\frac{\delta_{1}(z)-1}{\delta_{1}(z)+1}=\frac{1}{2}\left[b_{1} z+\left(b_{2}-\frac{b_{1}^{2}}{2}\right) z^{2}+\left(\frac{b_{1}^{3}}{2^{2}}-b_{1} b_{2}+b_{3}\right) z^{3}+\cdots\right]
$$

and following the same process

$$
\delta_{2}(\omega)=\frac{1+v(\omega)}{1-v(\omega)} \Longrightarrow v(\omega)=\frac{\delta_{2}(\omega)-1}{\delta_{2}(\omega)+1}=\frac{1}{2}\left[c_{1} \omega+\left(c_{2}-\frac{c_{1}^{2}}{2}\right) \omega^{2}+\left(\frac{c_{1}^{3}}{2^{2}}-c_{1} c_{2}+c_{3}\right) \omega^{3}+\cdots\right]
$$

Substituting (18) into (16) as expressed by (4) we get

$$
\begin{aligned}
\phi(u(z))=1 & +\frac{1}{2} \beta_{1} b_{1} z+\frac{1}{2}\left[\beta_{1}\left(b_{2}-\frac{b_{1}^{2}}{2}\right)+\frac{1}{2} \beta_{2} b_{1}^{2}\right] z^{2} \\
& +\frac{1}{2}\left[\beta_{1}\left(\frac{b_{1}^{3}}{2^{2}}-b_{1} b_{2}+b_{3}\right)+\beta_{2} b_{1}\left(b_{2}-\frac{b_{1}^{2}}{2}\right)+\frac{1}{4} \beta_{3} b_{1}^{3}\right] z^{3}+\cdots,
\end{aligned}
$$

and substituting (19) into (17) as expressed by (4) we get

$$
\begin{aligned}
\phi(v(\omega))=1 & +\frac{1}{2} \beta_{1} c_{1} \omega+\frac{1}{2}\left[\beta_{1}\left(c_{2}-\frac{c_{1}^{2}}{2}\right)+\frac{1}{2} \beta_{2} c_{1}^{2}\right] \omega^{2} \\
& +\frac{1}{2}\left[\beta_{1}\left(\frac{c_{1}^{3}}{2^{2}}-c_{1} c_{2}+c_{3}\right)+\beta_{2} c_{1}\left(c_{2}-\frac{c_{1}^{2}}{2}\right)+\frac{1}{4} \beta_{3} c_{1}^{3}\right] \omega^{3}+\cdots
\end{aligned}
$$

Now comparing the coefficients in (14) and (20) we get

$$
\begin{gathered}
\frac{[2]_{q}\left(1+[1]_{q} \lambda\right) a_{2}}{\tau}=\frac{\beta_{1} b_{1}}{2}, \\
\frac{[3]_{q}\left(1+[2]_{q} \lambda\right) a_{3}}{\tau}=\frac{1}{2}\left[\beta_{1}\left(b_{2}-\frac{b_{1}^{2}}{2}\right)+\frac{1}{2} \beta_{2} b_{1}^{2}\right],
\end{gathered}
$$

and comparing the coefficients in (15) and (21) gives

$$
\begin{gathered}
-\frac{[2]_{q}\left(1+\lambda[1]_{q}\right) a_{2}}{\tau}=\frac{\beta_{1} c_{1}}{2}, \\
\frac{[3]_{q}\left(1+[2]_{q} \lambda\right)\left(2 a_{2}^{2}-a_{3}\right)}{\tau}=\frac{1}{2}\left[\beta_{1}\left(c_{2}-\frac{c_{1}^{2}}{2}\right)+\frac{1}{2} \beta_{2} c_{1}^{2}\right] .
\end{gathered}
$$

Now adding (22) and (24) and simplifying we get

$$
b_{1}=-c_{1} \quad \text { and } \quad b_{1}^{2}=c_{1}^{2} .
$$

Also from (22) and (24) we get

$$
8[2]_{q}^{2}\left(1+[1]_{q} \lambda\right)^{2} a_{2}^{2}=\tau^{2} \beta_{1}^{2}\left(b_{1}^{2}+c_{1}^{2}\right),
$$

and adding (23) and (25) and using (26) we get

$$
4[3]_{q}\left(1+[2]_{q} \lambda\right) a_{2}^{2}=\tau \beta_{1}\left(b_{2}+c_{2}\right)-\tau b_{1}^{2}\left(\beta_{1}-\beta_{2}\right) .
$$


From (27) and using (26) we get

$$
b_{1}^{2}=\frac{4[2]_{q}^{2}\left(1+[1]_{q} \lambda\right)^{2} a_{2}^{2}}{\tau^{2} \beta_{1}^{2}} .
$$

So that by substituting for $b_{1}^{2}$ in (28) we get

$$
a_{2}^{2}=\frac{\tau^{2} \beta_{1}^{3}\left(b_{2}+c_{2}\right)}{4\left\{\tau \beta_{1}^{2}[3]_{q}\left(1+[2]_{q} \lambda\right)+[2]_{q}^{2}\left(1+[1]_{q} \lambda\right)^{2}\left(\beta_{1}-\beta_{2}\right)\right\}},
$$

and applying Lemma 2 gives (10).

Again by subtracting (23) from (25), using (26) and simplifying we get

$$
a_{3}=a_{2}^{2}+\frac{\tau \beta_{1}\left(b_{2}-c_{2}\right)}{4[3]_{q}\left(1+[2]_{q} \lambda\right)} .
$$

Thus, from (27), using (26) and simplifying we get

$$
a_{3}=\frac{\tau^{2} \beta_{1}^{2} b_{1}^{2}}{4[2]_{q}^{2}\left(1+[1]_{q} \lambda\right)^{2}}+\frac{\tau \beta_{1}\left(b_{2}-c_{2}\right)}{4[3]_{q}\left(1+[2]_{q} \lambda\right)}
$$

and applying Lemma 2 gives (11).

Let $q \uparrow 1$, then Theorem 4 becomes

Corollary 5. Let $f(z) \in \mathcal{B}_{q}(\tau, \lambda, \phi)$, then as $q \uparrow 1$,

$$
\begin{aligned}
& \left|a_{2}\right| \leq \frac{|\tau| \beta_{1}^{3 / 2}}{\sqrt{\left|\tau[3]_{q} \beta_{1}^{2}+[2]_{q}^{2}\left(\beta_{1}-\beta_{2}\right)\right|}}, \\
& \left|a_{3}\right| \leq \frac{|\tau|^{2} \beta_{1}^{2}}{[2]_{q}^{2}}+\frac{|\tau| \beta_{1}}{[3]_{q}} .
\end{aligned}
$$

which is the result of Srivastava and Bansal [10].

Theorem 6 ( Fekete-Szegö Estimate, $\varrho \in \mathbb{R}$ ). If $f \in \mathcal{B}_{q}(\tau, \lambda, \phi)$ and $\varrho \in \mathbb{R}$, then

$$
\left|a_{3}-\varrho a_{2}^{2}\right| \leq \begin{cases}\frac{|\tau| \beta_{1}}{[3]_{q}\left(1+[2]_{q} \lambda\right)} & \text { for } 0 \leq|h(\varrho)| \leq \frac{1}{[3]_{q}\left(1+[2]_{q} \lambda\right)} \\ |\tau| \beta_{1}|h(\rho)| & \text { for }|h(\varrho)| \geq \frac{1}{[3]_{q}\left(1+[2]_{q} \lambda\right)}\end{cases}
$$

where

$$
h(\varrho)=\frac{\tau \beta_{1}^{2}(1-\varrho)}{\left\{\tau \beta_{1}^{2}[3]_{q}\left(1+[2]_{q} \lambda\right)+[2]_{q}^{2}\left(1+[1]_{q} \lambda\right)^{2}\left(\beta_{1}-\beta_{2}\right)\right\}} .
$$

Proof. From (30) and (31),

$$
\begin{aligned}
\left|a_{3}-\varrho a_{2}^{2}\right| & =\left|\frac{\tau \beta_{1}\left(b_{2}-c_{2}\right)}{4[3]_{q}\left(1+[2]_{q} \lambda\right)}+(1-\varrho) a_{2}^{2}\right| \\
& =\left|\frac{\tau \beta_{1}}{4}\left\{\frac{\left(b_{2}-c_{2}\right)}{[3]_{q}\left(1+[2]_{q} \lambda\right)}+\left(b_{2}+c_{2}\right) h(\varrho)\right\}\right|,
\end{aligned}
$$

where $h(\varrho)$ is given in (33), so that by applying triangle inequality, (4), Lemma 2 and simplifying complete the proof. 
Theorem 7 ( Fekete-Szegö Estimate, $\rho \in \mathbb{C}$ ). If $f \in \mathcal{B}_{q}(\tau, \lambda, \phi)$ and $\rho \in \mathbb{C}$, then

$$
\left|a_{3}-\rho a_{2}^{2}\right| \leq\left\{\begin{aligned}
\frac{|\tau| \beta_{1}}{[3]_{q}\left(1+[2]_{q} \lambda\right)} \text { for }|1-\rho| \in[0, \xi) ; \\
\frac{\beta_{1}^{2}|\tau|^{2}}{[2]_{q}^{2}\left(1+[1]_{q} \lambda\right)^{2}}|1-\rho| \text { for }|1-\rho| \in[\xi, \infty),
\end{aligned}\right.
$$

where

$$
\xi=\frac{[2]_{q}^{2}\left(1+[1]_{q} \lambda\right)^{2}}{|\tau| \beta_{1}[3]_{q}\left(1+[2]_{q} \lambda\right)}
$$

Proof. From (27) and (31) and using (26),

$$
a_{3}-\rho a_{2}^{2}=(1-\rho) \frac{\beta_{1}^{2} b_{1}^{2} \tau^{2}}{4[2]_{q}^{2}\left(1+[1]_{q} \lambda\right)^{2}}+\frac{\beta_{1} \tau\left(b_{2}-c_{2}\right)}{4[3]_{q}\left(1+[2]_{q} \lambda\right)} .
$$

From Lemma 3 and (26)

$$
b_{2}-c_{2}=\frac{1}{2}\left(4-b_{1}^{2}\right)(x-y),
$$

for some $x, y,|x| \leq 1,|y| \leq 1$ and $\left|b_{1}\right| \in[0,2]$. Thus using (36) in (35) simplifies to

$$
a_{3}-\rho a_{2}^{2}=(1-\rho) \frac{\beta_{1}^{2} b_{1}^{2} \tau^{2}}{4[2]_{q}^{2}\left(1+[1]_{q} \lambda\right)^{2}}+\frac{\beta_{1} \tau\left(4-b_{1}^{2}\right)}{8[3]_{q}\left(1+[2]_{q} \lambda\right)}(x-y) .
$$

For $\delta(z)=1+b_{1} z+b_{2} z^{2}+\cdots \in \mathcal{P},\left|b_{1}\right| \leq 2$ by Lemma 2. Letting $b=b_{1}$, we may assume without any restriction that $b \in[0,2]$. Now using triangle inequality, letting $X=|x| \leq 1$ and $Y=|y| \leq 1$, then we get

$$
\left|a_{3}-\rho a_{2}^{2}\right| \leq|1-\rho| \frac{\beta_{1}^{2} b^{2}|\tau|^{2}}{4[2]_{q}^{2}\left(1+[1]_{q} \lambda\right)^{2}}+\frac{\beta_{1}|\tau|\left(4-b^{2}\right)}{8[3]_{q}\left(1+[2]_{q} \lambda\right)}(X+Y)=H(X, Y)
$$

For $X, Y \in[0,1]$;

$$
\max \{H(X, Y)\}=H(1,1)=\frac{\beta_{1}^{2}|\tau|^{2}}{4[2]_{q}^{2}\left(1+[1]_{q} \lambda\right)^{2}}\left\{|1-\rho|-\frac{[2]_{q}^{2}\left(1+[1]_{q} \lambda\right)^{2}}{\beta_{1}|\tau|[3]_{q}\left(1+[2]_{q} \lambda\right)}\right\} b^{2}+\frac{\beta_{1}|\tau|}{[3]_{q}\left(1+[2]_{q} \lambda\right)}=G(b) .
$$

For $b \in[0,2]$

$$
G^{\prime}(b)=\frac{\beta_{1}^{2}|\tau|^{2}}{2[2]_{q}^{2}\left(1+[1]_{q} \lambda\right)^{2}}\left\{|1-\rho|-\frac{[2]_{q}^{2}\left(1+[1]_{q} \lambda\right)^{2}}{\beta_{1}|\tau|[3]_{q}\left(1+[2]_{q} \lambda\right)}\right\} b,
$$

which implies that there is a critical point at $G^{\prime}(b)=0$, that is at $b=0$. Hence for

$$
G^{\prime}(b)<0 ;|1-\rho| \in\left[0, \frac{[2]_{q}^{2}\left(1+[1]_{q} \lambda\right)^{2}}{\beta_{1}|\tau|[3]_{q}\left(1+[2]_{q} \lambda\right)}\right),
$$

thus, $G(b)$ is strictly a decreasing function of $|1-\rho|$, therefore from (3),

$$
\max \{G(b): b \in[0,2]\}=G(0)=\frac{\beta_{1}|\tau|}{[3]_{q}\left(1+[2]_{q} \lambda\right)} .
$$

Also for

$$
G^{\prime}(b) \geq 0 ;|1-\rho| \in\left[\frac{[2]_{q}^{2}\left(1+[1]_{q} \lambda\right)^{2}}{\beta_{1}|\tau|[3]_{q}\left(1+[2]_{q} \lambda\right)}, 0\right),
$$

thus, $G(b)$ is an increasing function of $|1-\rho|$, therefore from (3),

$$
\max \{G(b): b \in[0,2]\}=G(2)=\frac{|1-\rho| \beta_{1}^{2}|\tau|^{2}}{[2]_{q}^{2}\left(1+[1]_{q} \lambda\right)^{2}} .
$$


So that by putting the results together leads to (34).

\section{Conclusion}

In this work, we were able to establish the first two coefficient bounds and also solve the Fekete-Szegö problem for the class $\mathcal{B}_{q}(\tau, \lambda, \phi)$ of analytic and bi-univalent functions in $\mathcal{U}$. The results in the first theorem generalized that of Srivastava and Bansal [10].

Acknowledgments: The authors thank the referees for their valuable suggestions to improve the paper.

Author Contributions: All authors contributed equally to the writing of this paper. All authors read and approved the final manuscript.

Conflicts of Interest: "The authors declare no conflict of interest."

\section{References}

[1] Duren, P. L. (2001). Univalent Functions. Springer-Verlag Inc., New York.

[2] Ma, W.C. \& Minda, D. (1994). A Unified Treatment of some Special Classes of Univalent Functions. Proceedings of the International Conference on Complex Analysis at the Nankai Institute of Mathematics, Nankai University, Tianjin, China. 157-169.

[3] Fekete, M., \& Szegö, G. (1933). Eine Bemerkung über ungerade schlichte Funktionen. Journal of the London Mathematical Society, 1(2), 85-89.

[4] Mazi, E. P., \& Opoola, T. O. (2017). On some subclasses of bi-univalent functions associating pseudo-starlike functions with Sakaguchi type functions. General Mathematics, 25, 85-95.

[5] Mazi, E.P. \& Opoola, T.O. (2019). A newly defined subclass of bi-univalent functions satisfying subordinate conditions. Mathematica 61,(84)(2), 146-155.

[6] Lewin, M. (1967). On a coefficient problem for bi-univalent functions. Proceedings of the American Mathematical Society, 18, 63-68.

[7] Murugusundaramoorthy, G. \& Bulut, S. (2018). Bi-Bazilevič functions of complex order involving Ruscheweyh type $q$-difference operator. Annales Universitatis Paedagogicae Cracoviensis. Studia Mathematica, 17, 5-18.

[8] Bulut, S. (2017). Certain subclass of analytic and bi-univalent function involving the $q$-derivative operator. Communications de la Faculté des Sciences de l'Université d'Ankara. Séries A1, 66(1), 108-114.

[9] Shaba, T.G. (2020). On some new subclass of bi-univalent functions associated with the Opoola differential operator. Open Journal of Mathematical Analysis, 4(2), 74-79.

[10] Srivastava, H.M. \& Bansal, D. (2015). Coefficient estimates for a subclass of analytic and bi-univalent functions. Journal of Egyptian Mathematical Society, 23, 242-246.

[11] Srivastava, H.M., Mishra, A.K. \& Gochhayt, P. (2010). Certain subclasses of analytic and bi-univalent functions. Applied Mathematics Letters, 23, 1188-1192.

[12] Jackson, F.H. (1908). On $q$-functions and a certain difference operator. Transactions of the Royal Society of Edinburgh, $46(2), 64-72$.

[13] Aral, A., Gupta, V. \& Agarwal, R.P. (2013). Applications of q-Calculus in Operator Theory. Springer Science+Business Media, New York.

[14] Kac, V. \& Cheung, P. (2002). Quantum Calculus. Springer-Verlag Inc., New York.

[15] Grenander, U. \& Szegö, G. (1958). Toeplitz Form and Their Applications. California Monographs in Mathematical Sciences, University of California Press, Berkeley.

[16] Libera, R.J. \& Zlotkiewicz, E.J. (1982). Early coefficients of the inverse of a regular convex function. Proceedings of the American Mathematical Society, 85(2), 225-230.

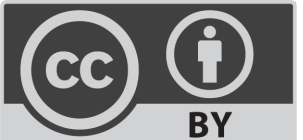

(C) 2021 by the authors; licensee PSRP, Lahore, Pakistan. This article is an open access article distributed under the terms and conditions of the Creative Commons Attribution (CC-BY) license (http://creativecommons.org/licenses/by/4.0/). 